\title{
Repérer les dynamiques de changement déjà présentes lors de demandes d'aide
}

\begin{abstract}
Abstrait
Faire une recherche sur la construction de l'expérience implique de travailler sur le mode même dont l'expérience se construit : dans son continuum, dans ses aspects micro-sociaux, dans ses transformations simultanées de l'action et de la pensée de l'action. Bref, il s'agit de construire la recherche, comme se construit l'expérience. C'est le parti pris de ce texte qui a pour objet plus particulier la première demande d'aide, enjeu pour tous les métiers de l'agir sur/avec/pour autrui. Dans l'intervention professionnelle la première demande adressée à l'intervenant est simplement considérée comme une prémisse de son intervention nécessaire pour établir le lien, mais qui n'est pas toujours constituée en ressource pour l'action auprès des personnes. Les matériaux des demandes d'aides recueillis dans le cadre d'un dispositif d'accès aux droits et de recours en santé mentale sont ici décryptés selon une approche d'analyse des activités pour repérer dès la première mise en contact les dynamiques déjà en cours, potentiellement mobilisables par les partenaires de l'échange. Les comportements révélateurs des dynamiques d'acteurs sont présents dans la manière de se présenter et de se positionner. Elles donnent à voir et à entendre les limites et les termes des compromis que les personnes en demande d'aide jugent possibles et acceptables. Considérer l'affirmation de soi, de sa volonté et de ses choix comme autant de dynamiques permet de mettre en mouvement les personnes et de transformer la situation dans laquelle elles se trouvent.
\end{abstract}

Mots-clés : analyse de l’activité, expérience, demande d'aide, dynamiques de changement.

\footnotetext{
* Chaire Unesco - ICP ; Consevatoire National des Arts et Métiers, Paris, France. Article soumis le 1 février 2021 ; accepté pour publication le 3 mars 2021.
} 


\title{
Identifying the Dynamics of Change Already Present when Applying for Aid
}

\begin{abstract}
Researching the construction of experience involves working on the very mode in which experience is built in its continuum, in its micro-social aspects, in its simultaneous transformations of action and thought of action. In short, it is a matter of building research as experience is built. It is the basis of this text that has as its particular subject the first request for help, an issue for all professions who act on/with others. In professional intervention, the first request to the intervener is simply considered to be a premise of his intervention necessary to establish the link, but which is not always constituted as a resource for action with individuals. The materials for requests for aid collected through a mental health access and recourse scheme are deciphered here using an activity analysis approach to identify from the first contact the dynamics already underway, potentially mobilized by the exchange partners. Behaviours that reveal the dynamics of actors are present in the way they present and position themselves. They show and hear the limits and terms of the compromises that those seeking help consider possible and acceptable. To consider self-assertion, one's will and one's choices, as dynamics allows people to move and transform the situation in which they find themselves.
\end{abstract}

Keywords: activity analysis, experience, request for help, dynamics of change.

\section{S'intéresser à la première demande d'aide adressée aux intervenants sociaux}

Dans l'intervention sociale et/ou de santé, la demande d'aide adressée à l'intervenant est habituellement considérée, sur le plan professionnel, davantage comme une prémisse de son intervention que comme une occasion d'apprentissage : la demande d'aide légitime l'intervention, permet d'établir une relation de confiance en rassurant le demandeur sur l'écoute et la disponibilité de l'intervenant. Elle est souvent jugée insuffisante en tant que telle pour comprendre, et donc agir.

Le plus souvent l'intervenant s'attache à entendre l'expression de la souffrance justifiant le recours à un tiers, notamment le vécu d'émotions négatives, vus par l'intervenant comme autant de causes sclérosant l'initiative du demandeur. La personne demandeuse est par ailleurs toujours suspecte d'attentes jugées illégitimes, notamment une réponse immédiate et magique à son problème.

Les travaux sociologiques et l'approche interactionniste rendent compte des contraintes sociales qui régissent l'expression de ces demandes dans des rapports asymétriques. Les personnes demandeuses se conforment à un registre jugé pertinent d'énonciation pour susciter l'attention et la réponse de l'accompagnant (Fassin 2000). Ces personnes demandeuses ont l'habitude de s'adresser à des professionnels ; elles partagent un même monde de significations mais à une place 
sociale différente, l'une censée posséder un savoir et un pouvoir, l'autre censée être incompétente et démunie. Chacune connaît la place qu'elle occupe dans le système social et agit en conséquence. Comme le dit Erving Goffman (1975) chacun connaît les deux rôles.

A rebours de ces constats, ce texte s'inscrit dans une double intention :

- Considérer ces premières demandes comme une étape essentielle dans la construction de la relation (cela suffit-il ?) entre demandeur et intervenant ;

- Analyser ce premier échange, à l'initiative du demandeur, comme riche de multiples messages sur son vécu de la situation et sur la dynamique de son itinéraire, éléments habituellement sous-estimés parce que peu conscientisés, pris en compte essentiellement sur le mode de l'intuition.

Dans une perspective d'analyse des activités (Barbier 2017) et d'optimisation des pratiques professionnelles, ne serait-il pas intéressant de s'interroger sur les activités et les positionnements des demandeurs manifestes dès ce premier appel, et ainsi de repérer les dynamiques déjà en cours, potentiellement mobilisables par les partenaires?

Ce travail a pour support les matériaux des demandes d'aides, lettres, mails, transcriptions d'échanges téléphoniques recueillis dans le cadre d'un dispositif d'accès aux droits et de recours en santé mentale.

\section{Soutenir des démarches visant à 'faire valoir ses droits en santé mentale'}

Depuis quelques années, s'est mise en place dans le champ de la santé mentale une pratique de soutien et d'accès aux droits entre pairs (Dutoit, Saint Pé 2018). Cette pratique, la Pairadvocacy ${ }^{1}$, entraide entre pairs, s'est développée dans le cadre d'une association d'usagers en santé mentale. Les personnes bénévoles qui pratiquent la Pairadvocacy sont des personnes qui ont connu des expériences de discrimination similaires aux vécus des personnes qu'elles épaulent.

La particularité de cet accompagnement est qu'il repose sur un a priori d'accueil inconditionnel de la personne demandant de l'aide, afin de déjouer le discrédit qui s'attache à la parole des personnes considérées comme ayant des troubles de santé mentale, fréquentant assez souvent un service de psychiatrie. Il s'agit d'une aide à la délibération visant à permettre à ces personnes de connaître leurs droits et de pouvoir les utiliser dans leurs stratégies d'action.

Dans cette pratique, sans visée thérapeutique, les pair-aidants ne savent rien des personnes qui les sollicitent, hormis ce qu'elles veulent bien leur confier. Le vécu des personnes demandant de l'aide est décrit en termes d"impasse' : elles se disent incomprises et/ou peu écoutées, discréditées le plus souvent par l'étiquette' qui leur a été attribuée dans le monde de la psychiatrie.

\footnotetext{
${ }^{1}$ Le dispositif est labellisé sous le nom de Pairadvocacy, https://www.pairadvocacy.eu
} 


\section{Les verbatims choisis pour analyser la demande initiale d'aide}

Nous avons choisi de travailler sur la transcription de trois demandes d'aide permettant d'accéder à des éléments comparables dans des histoires pourtant très différentes.

- L'une concerne une femme de soixante-dix ans (madame G.), retraitée après une carrière professionnelle, et se retrouvant volontairement à la rue. La transcription est extraite d'un entretien téléphonique avec la pairadvocate, dont les interventions sont en italique.

- La seconde transcription concerne un homme de 40 ans (monsieur N.), travaillant en ESAT (Établissement de service d'aide par le travail) et craignant de perdre son emploi. Elle provient d'un mail.

- La troisième est une lettre adressée par un homme de 60 ans (monsieur J.-M.) qui habite habituellement à l'hôtel, a perdu sa carte bancaire, dort une nuit dans un sas de banque fracturé, est appréhendé par la police et se retrouve en psychiatrie.

\section{Madame G.}

J'habitais un appartement dans un immeuble social. Avec les voisins ce n'était pas simple. Moi je ne fais pourtant pas de bruit. On a dit que j'avais un syndrome ... un truc comme iogène...

Le syndrome de Diogène

Oui c'est ça, je n'suis jamais malade, je n'suis pas folle, j'ai ma tête Je suis à la retraite, j'ai travaillé à la CAF toute ma vie et j'ai toujours payé mon loyer, maintenant je suis à la rue depuis 9 mois.

Vous êtes dans un CHRS que s'est-il passé?

Je ne voulais pas être prise par les psy ils voulaient que j'aille en maison de retraite, ils sont venus chez moi, je suis partie

Il y a eu une curatelle provisoire...

La curatrice avait un double de mes clés et elle prenait tout mon courrier... Depuis votre arrivée au CHRS que s'est-il passé ?

J'ai un psy qui me comprend, il n'veut pas m'hospitaliser mais la curatrice veut me faire signer un dossier de surendettement pour $1000 \mathrm{E}$ de dette de loyer? il y aurait un jugement de curatelle en octobre. Je n'veux pas la voir cette curatrice je ne signerais pas...

\section{Monsieur N.}

Bonjour je m'appelle $\mathrm{N}$, je voudrais mettre un dispositif de protection pour avoir des droits en tant que personne handicapé psychique parce que je subis des actes discriminatoires dans mon ESAT. Ayant fait du droit lors de mes anciennes formations (niveau 3 BTS comptabilité et gestion des organisations), cela serait relativement simple de construire mes droits avec vous. De plus mon établissement ESAT possède 'un projet d'établissement' (droit de notre ESAT) qui énumère et donne des droits aux personnes comme moi. 
Pour arriver là où j'en suis aujourd'hui j'ai lutté ! Je possède une RQTH que j'ai acquise parce que j'ai assisté à la commission de celle-ci. Je me suis sorti du dispositif médical (HP) il y a quelques années maintenant. Je travaille en ESAT qui est une structure médico-sociale avec un accompagnement en principe. Pourquoi vouloir créer de la désocialisation qui n'a pas lieu d'être ! Je n'ai pas de problème, on m'en crée et je serai là pour tout vous expliquer et pour tout vous énumérer.

J'ai besoin de construire et travailler concernant ce travail que je n'ai absolument pas envie de quitter! Aucune poursuite ou décision en justice n'est déclarée, mais j'ai eu une amorce négative de ma hiérarchie qui me laisse perplexe. Vous savez que pour les personnes comme moi ça va très vite la désocialisation non justifiée, et c'est pour cela que je vous sollicite afin faire valoir mes droits.

Monsieur J.-M.

Après la perte de ma carte bancaire (je perçois le minimum vieillesse) je me suis retrouvé à la rue. Même avec ma carte d'identité et mon numéro de compte postal, on ne m'a pas permis de retirer de l'argent. J'en étais donc réduit à la mendicité et à dormir où je pouvais. Une nuit de février, vers deux heures du matin, je ne savais pas où dormir et j'ai vu un gymnase. Dans les gymnases il y a des hébergements Grand-Froid. Ce gymnase était fermé et à côté il y avait un local de banque avec la porte ouverte et cela était éclairé et chauffé. La porte du local avait été ouverte par des cambrioleurs. Mais cette nuit-là il faisait très froid et nécessité faisant loi, j'ai pensé que j'allais pouvoir rester au chaud jusqu'au lever du jour.

Mais la police est venue m'interpeler à six heures du matin pensant que j'étais l'auteur du cambriolage.

Un avocat à fait dériver la loi vers la psychiatrie. Depuis je suis enfermé en psychiatrie, sous le régime de la contrainte sans consentement. Je n'ai plus d'avocat pour être présenté au Juge des libertés. Je suis né en 1950 et en psychiatrie ils ne veulent plus que je sorte, alors qu'après ma sortie je peux être hébergée chez une amie. Ils veulent que j'aille en maison de retraite. Je pense que je suis trop jeune pour aller en maison de retraite, surtout qu'ils veulent que je sois mis en incapacité (curatelle renforcée). Il ne me resterait que 50 euros par mois, à peine de quoi m'acheter du tabac et rien pour des vêtements, chaussures, journaux, livres. Je formule donc une requête envers vous.

Je vous adresse l'expression de mes salutations distinguées et vous remercie à l'avance.

PS excusez-moi pour les fautes d'orthographe, de grammaire et de syntaxe.

Suite à ce courrier, le premier contact téléphonique fait apparaître que le mode habituel de vie de la personne est l'hôtel ; il se présente d'ailleurs comme un grand voyageur. Il indique par ailleurs avoir rompu avec sa famille à l'âge de 18 ans pour faire la route de Katmandou. 


\section{La présentation de soi par les personnes demandeuses}

On note trois caractéristiques communes aux récits recueillis lors du premier contact :

1. Les personnes mettent en avant leurs qualités pour la vie sociale ou leur adaptation : Madame G. ne fait «pas de bruit », elle a " toute sa tête »; elle a un statut social de retraitée, qui a travaillé et en plus à la CAF (Caisse d'Allocations Familiales) ; elle remplit ses obligations auprès de son bailleur ; elle est simplement partie de son domicile. Monsieur N. se présente d'emblée par son ancien statut de travailleur et par son niveau d'étude. Il a voulu être en Etablissement de Services d'Aide par le Travail (ESAT), s'est battu pour cela et n'a pas de problème. Monsieur J.-M. maîtrise le fonctionnement des dispositifs sociaux, d'autant plus qu'il a toujours cherché à les déjouer.

2. Les personnes se présentent en utilisant des catégories sociales légitimes dans le monde de la santé mentale : utilisation du mot syndrome, référence aux droits des personnes handicapées, référence à l'existence d'un projet d'établissement régissant ces droits, connaissance des compétences du juge des libertés et de la loi régissant la contrainte en psychiatrie.

3. Elles souffrent néanmoins d'actions négatives de la part de personnes disposant d'un pouvoir sur elles dans la situation dans laquelle elles se trouvent. Pour madame G., « les psy » veulent l'envoyer en maison de retraite et la « curatrice » rentre chez elle et « prend son courrier ». Monsieur N. quant à lui se sent menacé par « la hiérarchie » de l'ESAT (sans nommer expressément à quel niveau : c'est plutôt le fait même d'être travailleur handicapé qui le met en position possible d'être victime d'une "désocialisation non justifiée ». Monsieur J.-M. se plaint d'être placé sous curatelle et contraint à intégrer une maison de retraite alors qu'il a une solution de sortie.

On le voit, on se trouve en présence de 'réactions' à la situation qui, de leur point de vue, leur est faite par d'autres acteurs ayant un pouvoir institutionnel. Ces réactions sont à la fois personnelles, élaborées, très significatives des interprétations qu'ils construisent de et dans la situation, et dont l'intervenant se trouve de fait être destinataire, même s'il n'est pas en mesure d'en tirer immédiatement profit pour son intervention.

\section{L'analyse des demandes}

La demande d'aide, à l'analyse, fait apparaître, la présence de trois types d'informations significatives pour comprendre la demande :

- Ce qui 'met en mouvement' la personne demandeuse : le changement de sa situation,

- 'Ce qu'elle voudrait obtenir,' en lien avec son interprétation de sa situation, 
- La « 'part de soi' qu'elle revendique » dans la recherche d'une solution et dans la relation d'aide.

«Le changement de situation : ce qui fait rupture ». Pour Madame G. la stabilité de sa situation antérieure est marquée par l'usage de l'imparfait, temps long de la stabilité, le passé composé est celui de l'événement. Le présent « je suis à la rue depuis six mois » est en rupture avec le temps long de la stabilité.

Pour monsieur N., ce qui fait rupture c'est une question qui surgit, sous une forme abstraite, c'est-à-dire, détachée du concret des événements qui la précédent : «Pourquoi vouloir créer de la désocialisation qui n’a pas lieu d'être ! » mais qui le concerne directement, c'est bien de sa désocialisation dont il est question.

Pour monsieur J.-M., le changement de situation est entrainé par la perte de sa carte bancaire qui signifie la perte de ses moyens de vivre de façon autonome.

"Ce que la personne veut obtenir, en lien avec son interprétation de la situation ". Si les trois personnes sollicitent d'elles-mêmes le soutien, elles évoquent déjà là où elles veulent aller ou comment elles veulent $\mathrm{y}$ aller. Pour Madame G., pas de reddition à demande de la curatrice. Pour monsieur N., construire la défense de ses droits avec l'intervenant. Pour monsieur J.-M. retrouver sa liberté d'aller et venir.

Cette volonté est cohérente avec l'interprétation qu'elles font de la situation.

Madame G. a choisi de partir de chez elle : c'est sa façon de répondre aux pressions et aux intrusions éprouvées ; elle ne s'est pas retrouvée à la rue, puis au centre d'hébergement d'urgence (CHRS), par choix, mais par conséquence de ces pressions et intrusions, conséquence qui l'oblige à endosser une image négative, de personne "prise en charge ", démunie, voire pauvre. Pour monsieur N., la menace de « désocialisation non justifiée » est réelle : en rappelant son statut de patient et d'handicapé psychique, il relie les difficultés contre lesquelles il s'est battu et ce nouvel épisode dans une même série d'événements qui ont toujours la même cause. Pour monsieur J.-M., ce sont les institutions qu'il a cherché à fuir toute sa vie qui le 'rattrapent' à 60 ans pour le priver de sa liberté.

On le voit, dans les trois cas les systèmes d'interprétation institutionnels et personnels sont radicalement opposés.

« La 'part de soi' qu'elles veulent faire reconnaître par les autres acteurs ». Madame G. reste combative, ne veut pas perdre la face : accepter de voir cette curatrice c'est annuler son acte de résistance : " partir de chez elle », s'échapper, donc elle refuse « Je n'veux pas la voir cette curatrice je ne signerai pas ».

Monsieur N. veut faire reconnaître sa capacité à contribuer à sa propre défense : «cela serait relativement simple de construire mes droits avec vous ». Il se revendique comme un combattant, fier d'avoir lutté, d'être sorti de l'Hôpital Psychiatrique et d'avoir obtenu de son statut de travailleur handicapé (RQTH) dont on comprend qu'il l'a obtenu en se défendant devant la commission qui l'accorde.

Monsieur J.-M. sollicite le respect de son choix de vie opposé à ce que représente habituellement la maison de retraite. 
Malgré les difficultés, madame G. et monsieur N., monsieur J.-M. manifestent d'emblée une confiance en eux dans l'action. Madame G. dit qu'elle n'est pas responsable de la dette de loyer et donc qu'elle ne rencontrera pas la curatrice ; elle laisse penser qu'elle ne transigera avec cette curatrice qu'à la condition de ne plus la rendre responsable des $1000 \mathrm{E}$ de dettes. Le recours à une commission d'endettement est pour elle un vécu humiliant. Monsieur N. ne lâchera pas sur ses droits de personne handicapée psychique. Il ne se positionne pas en tant que personne ayant un conflit de travail, mais comme un ayant droit du fait de son statut dans ce type d'institution. Monsieur J.-M. se positionne en citoyen assumant ses choix de vie.

Les trois personnes affichent une position 'haute' dès le premier contact, position qui peut être lue comme un obstacle, mais aussi comme l'affirmation d'un potentiel d'engagement dans la sortie de la situation dans laquelle ils se trouvent.

\section{Des comportements révélateurs de dynamiques d'acteurs d'ores et déjà présentes}

Ces comportements sont révélateurs de dynamiques déjà présentes dans la situation. Elles ont deux faces :

- L'affirmation personnelle ;

- La contrainte sociale.

Dans le cas de madame G., on se trouve en présence d'une attitude de restauration de soi, caractérisée par le retour à une situation antérieure préservant la représentation qu'elle s'est toujours faite d'elle-même tout en étant en conformité avec les attentes sociales.

Cette dynamique sera confirmée par l'issue de son itinéraire : Madame G. ne pouvait pas reconnaître cette dette de mille euros et donc voir la curatrice pour signer un dossier de surendettement qui aurait signifié sa responsabilité. C'est donc lorsque le bailleur a reconnu que ces mille euros correspondaient aux neuf mois durant lesquels elle avait été à la rue, qu'une négociation a pu s'engager. Un compromis a même finalement été trouvé pour annuler cette dette, lui permettant de récupérer son logement et, élément décisif, d'échapper à une curatelle définitive. La contrepartie est l'acceptation d'un suivi psychiatrique et social. Ce suivi est accepté par Madame G. comme une concession moindre puisque le psychiatre est apparu comme bienveillant dans la relation et actif dans la résolution de sa situation, notamment dans la réassurance du bailleur. La situation se résout parce que Madame G. tient bon.

Dans le cas de monsieur N., on se trouve en présence d'une attitude d'endossement de l'étiquette qui lui donne une énergie personnelle adossée au poids social pris par la catégorie des personnes handicapées.

Dans la suite donnée à son problème, monsieur N. ne lâchera pas non plus son positionnement arrimé fortement à la légitimité de son statut de personne 
handicapée psychique en RQTH (Reconnaissance Travailleur handicapée), obtenu de haute lutte. C'est donc en endossant cette étiquette jugée pourtant négative par d'autres et en revendiquant les droits attachés à son statut qu'il va obtenir une place dans la confrontation qui aura lieu auprès du Directeur de son ESAT et de son chef d'atelier. Ainsi un simple accompagnement lors de cet entretien lui a permis de replacer le problème de travail dans le cadre du droit des personnes handicapées, de l'engagement de l'établissement, du respect et de la prise en compte de ce statut dans la relation de travail. Ce cadre posé, la « hiérarchie » de l'ESAT a clarifié ses attentes en termes de travail. Rassuré, monsieur N. a pu progresser dans la relation salariale établie dans le travail à effectuer.

Dans le cas de monsieur J.-M., on se trouve dans une attitude mêlant déni, qui lui donne de la force personnelle, et concession aux contraintes sociales pour autant qu'elles ne remettent pas en cause la face qu'il veut garder à ses propres yeux et devant autrui. Monsieur J.-M. reste de très long mois en psychiatrie, refusant la proposition d'une maison de retraite. Il affirme son identité de « baroudeur » en enfreignant le plus souvent possible le règlement ce qui lui vaut des « punitions » restreignant sa liberté d'aller et venir dans l'enceinte de l'hôpital. On l'oblige à visiter une maison de retraite qu'il déteste, mais s'ouvre alors une réelle possibilité d'échappée à l'enfermement psychiatrique qu'il subit depuis si longtemps. Il pourrait essayer d'implanter dans ce cadre de vie un mode de vie compatible avec sa liberté d'aller et venir. Certes, il est soumis à l'obligation d'être présent au repas et de rentrer le soir aux heures prescrites (qu'il appelle le 'couvrefeu'), mais le reste du temps il peut vaquer à ses occupations et puis, ce n'est pas la prison, il garde l'espoir que peut-être un jour il aura la force de partir. En fait, monsieur J.-M. est depuis de nombreuses années en maison de retraite, toujours à la limite de l'insubordination. Le seul combat qui reste engagé, même s'il reste sans succès, concerne sa mise sous curatelle, frein à son désir revendiqué de partir ailleurs.

Les repérages de ces trois attitudes, qui s'est fait à l'intuition par les intervenants, a permis la mise en mouvement des autres acteurs dans des situations apparemment bloquées sur la conformité au social : les rapports de place se sont modifiés dans la situation. Il autorise l'hypothèse de l'intérêt de construire des outils de repérage des attitudes des demandeurs dès la première demande.

\section{La réponse professionnelle : un essai de prise en compte des éléments identifiés}

Ces demandes sont donc reçues par les personnes pairadvocates. Centré sur une écoute active, la pratique du pairadvocate consiste à vouloir prendre en compte ce que désire la personne. L'objectif étant que la personne demandeuse devienne son propre défenseur en construisant une stratégie pour résoudre son problème avec le 
soutien du tiers accompagnant. Une partie de son travail de soutien consiste à permettre à ces personnes d'explorer ce qui fait problème pour elle dans la situation, de repérer avec elle les différents éléments en jeu, et la place qu'ellemême occupe dans la situation. Ainsi, être sensible aux éléments dynamiques présents dans cette demande s'impose à ces accompagnants pour faire bouger les rapports entre les acteurs/trices identifié.e.s, tout en restant un exercice difficile.

L'hypothèse de travail de l'accompagnant est qu'il est possible pour la personne concernée de passer d'une vision où elle est à la fois cause et victime, responsable de la situation et contrainte à agir sur elle-même pour pouvoir la résoudre, à une vision stratégique fondée sur les intérêts et les potentiels de tous les acteurs présents dans la situation. Il s'agit donc notamment de construire la situation comme dynamique.

\section{La construction professionnelle d'un outil pour approfondir la première demande}

Le premier contact est donc un moment classique d'accroche de la relation ; mais bien plus, pour les pairadvocates, il contient des éléments donnés par la personne elle-même en cohérence avec ce qu'elle est et ses enjeux de vie, éléments faisant partie intégrante de la démarche de résolution de son problème. A partir de ce constat et sur les acquis des expériences d'accompagnement élaborées au fil des années, un effort de modélisation a été fait, notamment dans le souci de former de nouveaux praticiens.

Il devenait possible d'essayer d'élaborer un outil prenant en compte à la fois :

- Ce que permettait l'utilisation d'une écoute active par le pairadvocate.

- L'accompagnement des interactions entre personne demandeuse/pairadvocate, à l'aide d'une grille de questionnement, appelée « grille de construction de la situation problème ».

L'écoute active consiste à attirer l'attention de l'écoutant sur les éléments singuliers présents dans la demande d'aide : les mots et expressions significatifs. Ici, il est fait référence au concept d'indexicalité utilisé en ethnométhodologie : "Bien qu'un mot ait une signification trans-situationnelle, il a également une signification distincte dans toute situation particulière dans laquelle il est utilisé » (Coulon 1987 : 29). Il s'agit de repérer la singularité des mots et des expressions, leur récurrence, l'univers sémantique auxquels ils renvoient, leur cohérence au regard de l'intention du locuteur, le sens que la personne leur donne pour donner à comprendre sa pensée, aux autres comme à soi-même.

La grille de description de la situation-problème est conçue comme une déconstruction/reconstruction, en posant un certain nombre de questions visant à établir des faits. Cette description est réalisée en trois étapes : 
- Une première étape « descriptive » consiste à établir les éléments qui composent la situation : les lieux, les moments, les acteurs/trices, et la place de la personne demandeuse.

- Une seconde étape, " compréhensive », consiste à établir des liens entre ces éléments il s'agit de : qui dit le problème, quelles interprétations en font les autres acteurs?

- Une troisième étape, plus « argumentative », consiste à formaliser les enjeux d'acteurs, en vue d'énoncer les leviers pour agir et de construire une stratégie pour intervenir : quelles sont les personnes à interpeller, les arguments à utiliser selon les attentes et les enjeux des différents acteurs/trices et selon la place sociale qu'elles ou qu'ils occupent.

Cet outil est mis constamment à l'épreuve de la pratique. Pour exemple, lors d'une formation réalisée auprès de juristes sourdes s'exprimant en langue des signes et voulant pratiquer la pairadvocacy dans une permanence spécifiquement réservée aux personnes sourdes, l'outil s'est enrichi : la construction de la situation passant uniquement par l'oralité pouvait mettre en difficulté ce public et appauvrir l'expression personnelle. Les juristes pairadvocates en formation ont proposé une modélisation visuelle de la situation-problème, ensuite étendue à l'ensemble des publics. Cet outil est donc toujours en construction.

\section{Des enjeux plus larges}

Prendre en compte la première demande : un enjeu dans tous les métiers de l'agir sur/avec/pour autrui. Dans ces différents métiers se pose la question de l'interaction à réussir pour que les propositions faites par les professionnels puissent être investies par le public. Faute de quoi rien ne se passera. Dans ces métiers, c'est d'ajustement à l'autre dont il est question.

Dans une précédente recherche dans le champ du handicap rare (Dutoit et all. 2018) nous avons repéré la notion de «propositions d'activité », c'est-à-dire de propositions spécifiquement adressées au partenaire de l'échange : «ce qu'il est possible de faire ensemble est un compromis permanent entre ce qui est proposable et ce qui est acceptable, tour à tour, et réciproquement par les partenaires » (ibidem : 39).

Dans les premières demandes analysées, nous avons vu que les trois personnes donnaient à voir et à entendre dans les verbatims les contours des compromis qu'elles pouvaient envisager. Même si les limites et les termes de ces compromis possibles et acceptables ne sont pas conscients et qu'ils se manifestent le plus souvent en " actes ", ils sont alors donnés à lire dans la manière dont se présentent et se positionnent les personnes dès le premier contact. 
Ainsi posés, ces éléments influencent l'écoutant, notamment pour faire des propositions congruentes avec le cadre posé. Ce n'est bien sûr pas toujours le cas, notamment si l'échange ne s'engage pas dans une action conjointe analysable en termes de couplage d'activités (Barbier, Thievenaz 2017).

Dans les métiers de l'accompagnement, le couplage d'activité peut rendre compte de situations, où l'activité de l'un est indissociable de celle de l'autre, l'activité de l'un est subordonnée à l'activité de l'autre et vise et versa. L'activité d'aide peut être vue comme l'établissement d'une activité réciproque, et analysée notamment avec ces deux notions : propositions d'activité et couplage d'activités.

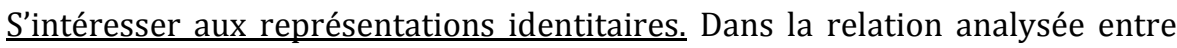
professionnels et public dans le champ du handicap rare, nous avons vu aussi que dans le jeu des propositions/négociations d'activité s'opéraient des représentations de soi comme sujet agissant.

Dans notre analyse, les revendications identitaires des personnes demandeuses sont fortement présentes ; sans doute sont-elles d'autant plus fortes qu'elles sont en réaction à un jugement social (Dutoit 2011). Identifier ces représentations dès ces premières demandes pourrait permettre de comprendre ces revendications dans les interactions en devenir entre partenaires de la relation et leur activation.

La notion de dynamique identitaire peut permettre de rendre compte de l'importance des jeux de reconnaissances réciproques, qui ont un impact sur l'engagement des sujets. De même, les notions de transaction sociale et " d'habilitation intersubjective » (Roelens $1998: 129$ ) permettent de lier transformations de représentations identitaires et transformations de places sociales.

La transaction suppose que les situations ne soient pas définies à l'avance, mais structurées par des tensions sur lesquelles il reste possible d'agir. Ainsi, nous pouvons considérer l'affirmation de soi, de sa volonté et de ses choix comme autant de dynamiques mettant en mouvement les jeux sociaux présents dans l'ensemble de la situation dans laquelle la personne se trouve.

Et ainsi, pouvoir repérer les dynamiques de changements présentes dans une situation d'appel à l'aide peut permettre de mettre en brèche des habitudes de pensé et d'action. Ces habitudes consistent souvent à déplorer la passivité et la mauvaise volonté des publics à changer de comportement.

Écouter ce que nous apprennent des premières demandes, c'est simultanément construire un lien de confiance avec son interlocuteur/trice et se laisser guider par les informations manifestes données par la personne, dans l'ici et le maintenant de la relation.

En somme, pour le chercheur comme pour le professionnel, utiliser toutes les ressources des situations, comme nous le propose François Jullien (2018) « Le propre de la ressource est qu'elle s'explore et qu'elle s'exploite ; et qu'on l'explore encore en l'exploitant » (ibidem : 23). 


\section{Bibliographie}

Barbier J.-M. (2017) Vocabulaire d'analyse de l'activité, Penser les conceptualisations ordinaires, Paris, Presses Universitaires de France.

Barbier J.-M., Thievenaz J. (2017) Agir pour, sur et avec autrui. Les couplages d'activités en question, Paris, L'Harmattan.

Coulon A. (1987) L'ethnométhodologie, Paris, Presses Universitaires de France, Coll. Que sais-je).

Dutoit M. (2011) Être vu, se voir, se donner à voir, Paris, L'Harmattan.

Dutoit M., Barbier J.-M., Rémery V., Arciniegas M. (2018) Apprendre d'une expérience rare, Paris, L'Harmattan.

Dutoit M., Saint Pé M.-C. (2018) Une expérience de réappropriation collective du droit in : M. Tremblay, V. Guerdan, M. Boukala, J.-P. Cobbaut, La Convention des Nations Unies Relative aux Droits des Personnes Handicapées : Une Nouvelle Éthique de la Citoyenneté, Wisconsin, USA, Deep Education Press : 148-161.

Fassin D. (2000) La Supplique. Stratégies rhétoriques et constructions identitaires dans les demandes d'aide d'urgence "Annales. Histoire, Sciences Sociales »55e Année, No. 5 (Sep.-Oct.) : 955-981, https://doi.org/10.3406/ahess.2000.279895

Jullien F. (2018) Ressources du christianisme, mais sans y entrer par la foi, Paris, Herne.

Goffman E. (1975) Stigmates, Les usages sociaux des handicaps, trad. A. Kihm, Paris, Minuit, Coll. Le sens commun.

Roelens N. (1998) Les transactions intersubjectives dans l'attribution de compétences in : M. F. Freynet, M. Blanc, G. Pineau, Les transactions aux frontières du social, Lyon, Chroniques Sociales : 121-136.

\section{Identifying the Dynamics of Change Already Present when Applying for Aid}

\section{Summary}

Researching the construction of experience involves working on the very mode in which experience is built in its continuum, in its micro-social aspects, in its simultaneous transformations of action and thought of action. In short, it is a matter of 
constructing a research project in the same way that experience is constructed. This is the starting point of this text that has as its particular subject the first request for help, an issue for all professions that act on/with/for others. In professional intervention, the first request to the intervener is simply considered to be a premise of his or her intervention, necessary to establish the link, but which is not always constituted as a resource for action with individuals.

The materials for requests for aid collected through a mental health access and recourse scheme are deciphered here using an activity analysis approach to identify, from the first contact, the dynamics already underway, potentially mobilized by the exchange partners involved in the exchange. Behaviours that reveal the dynamics of actors are present in the way they present and position themselves. They show and make audible the limits and terms of the compromises that those seeking help consider possible and acceptable. To consider self-assertion, one's will and one's choices as dynamics allows people to move and transform the situation in which they find themselves. The activity of helping can be seen as the establishment of a reciprocal activity and analysed with these two notions: activity proposals and coupling of activities. In the same way, the notion of identity dynamics can allow us to consider the importance of the interactions around mutual recognition, which have an impact on the commitment of the subjects, as does the notion of social transaction. These notions make it possible to link transformations of representations of identity and transformations of social status.

\section{Rozpoznanie dynamiki zmian obecnych w prośbie o pomoc}

\section{Streszczenie}

Prowadzenie badań nad konstruowaniem doświadczenia wymaga podjęcia pracy nad sposobami jego budowania, widocznymi w jego przebiegu, w szczegółowych aspektach mikrospołecznych, w równoczesnych transformacjach działania i myślenia o nim. Krótko mówiąc, chodzi o konstruowanie badań o tym, jak konstruowane jest doświadczenie. Jest to celem tego tekstu. Jego przedmiotem szczególnym jest pierwsza prośba o pomoc, która stanowi wyzwanie dla przedstawicieli wszystkich zawodów, którzy działają z/dla/wobec drugiego. W oddziaływaniu profesjonalnym pierwsza prośba o pomoc, skierowana do interweniującego, jest na ogół postrzegana jako przesłanka do podjęcia interwencji, niezbędnej do nawiązania więzi, ale nie zawsze stanowi podstawę do podjęcia działania.

Materiały dotyczące prośby o pomoc zostały zebrane w ramach systemu dostępu do praw i środków ochrony zdrowia psychicznego. W celu zidentyfikowania już trwającej od pierwszego kontaktu dynamiki potencjalnie mobilizującej partnerów wymiany dokonano ich analizy zgodnie z podejściem analizy aktywności. Zachowania ujawniające dynamikę aktorów widoczne są w sposobie prezentacji i usytuowania. Umożliwiają dostrzeżenie i „usłyszenie” granic oraz warunków kompromisu, które osoby proszące o pomoc uznają za możliwe do zaakceptowania. Uznanie, że samoafirmacja oraz chęć wolnego wyboru uruchamiają dynamikę i pozwalają ludziom 
na przekształcenie sytuacji, w której się znajdują. Aktywność pomocy może być postrzegana jako opracowanie wzajemnego oddziaływania i analizowania, w szczególności za pomocą dwóch kategorii pojęć: propozycji aktywności i ich wzajemnego powiązania/sprzężenia. Podobnie pojęcie dynamiki tożsamości może pozwolić na uwzględnienie znaczenia gier/związków wzajemnego uznania, które mają wpływ na zaangażowanie podmiotów, takich jak „transakcja społeczna”. Pozwalają one wiązać transformacje reprezentacji tożsamościowych z transformacjami usytuowania społecznego.

\section{Pour citer cet article}

Dutoit M. (2021) Repérer les dynamiques de changement déjà présentes lors de demandes d'aide, „Nauki o Wychowaniu. Studia Interdyscyplinarne” 2(13), 109-123, https://doi.org/10.18778/2450-4491.13.08 